\title{
A Minimalist Approach to Remote Attestation
}

\author{
Aurélien Francillon \\ Networking and Security Department, Eurecom \\ Sophia-Antipolis, France \\ aurelien.francillon@eurecom.fr
}

\author{
Quan Nguyen Kasper B. Rasmussen Gene Tsudik \\ Computer Science Department, UC Irvine \\ Irvine, CA, USA \\ quann1@uci.edu, kbrasmus@ics.uci.edu,gts@uci.edu
}

\begin{abstract}
Embedded computing devices increasingly permeate many aspects of modern life: from medical to automotive, from building and factory automation to weapons, from critical infrastructures to home entertainment. Despite their specialized nature as well as limited resources and connectivity, these devices are now becoming an increasingly popular and attractive target for attacks, especially, malware infections. A number of approaches have been suggested to detect and/or mitigate such attacks. They vary greatly in terms of application generality and underlying assumptions. However, one common theme is the need for Remote Attestation, a distinct security service that allows a trusted party (verifier) to check the internal state of a remote untrusted embedded device (prover). Many prior methods assume some form of trusted hardware on the prover, which is not a good option for small and low-end embedded devices. To this end, we investigate the feasibility of Remote Attestation without trusted hardware.

This paper provides a systematic treatment of Remote Attestation, starting with a precise definition of the desired service and proceeding to its systematic deconstruction into necessary and sufficient properties. Next, these are mapped into a minimal collection of hardware and software components that result in secure Remote Attestation. One distinguishing feature of this line of research is the need to prove (or, at least argue for) architectural minimality - an aspect rarely encountered in security research. This work also provides a promising platform for attaining more advanced security services and guarantees.
\end{abstract}

\section{INTRODUCTION}

"Embedded systems" is a broad notion that encompasses many kinds of specialized computing devices that vary greatly in terms of resources and intended purposes. Unlike generalpurpose computers that, for decades, have been the primary attack victims, embedded systems have been targeted only relatively recently. The Stuxnet [8] incident pointedly demonstrated the impressive scope and impact of malware on embedded devices. Stuxnet specifically targeted Programmable Logic Controllers (PLC) in industrial control systems. By modifying PLC control parameters, it ostensibly caused some serious physical damage.

Stuxnet should be viewed as both an example, a warning and a preview of coming attractions. It epitomizes the power and amplification factor of remote malware attacks, i.e., those not requiring direct access to victim devices. With growing presence and proliferation of networked embedded devices into many

Aurélien Francillon was supported by the European Commission within the STREP TAMPRES project. The views and conclusions contained herein are those of the authors and should not be interpreted as representing the official policies or endorsement of this project or the European Commission. spheres of life, (e.g., automation of homes, factories, office buildings as well as automotive, aerospace and public utilities sectors), remote malware attacks have become a clear and present danger. This motivates the need for countermeasures, a number of which have been proposed by the research community and some have been implemented by manufacturers. One common theme among them is Remote Attestation - a security service that involves verification of internal state of a remote embedded device. Although Remote Attestation is not a panacea, it should ideally allow for efficient and accurate detection of remote software attacks.

Prior research results have underscored the difficulty of the problem. We believe that, although ad hoc or specialized solutions might work in the near term, or for a narrow range of devices, only systematic approaches to Remote Attestation that offer concrete security guarantees are likely to prove effective in the long run. This assertion forms the premise for the work described in this paper.

We use the term Remote Attestation to denote attestation performed across a network. In this setting, software attestation techniques [13], [16], [17], [27]-[31] are not applicable, since software attestation can be secure only if the verifier communicates directly to the prover, with no intermediate hops [4]. At the other end of the solution spectrum are techniques based on secure hardware components, such as TPMs [35] or secure co-processors [33]. However, they represent a significant cost barrier for low-end embedded devices. We believe that the promise lies in a careful analysis of Remote Attestation as a distinct security service, including systematic identification of its necessary and sufficient components. This should ideally result in the design of a generic and practical embedded system architecture for Remote Attestation.

In this paper, we follow the above path: starting with the definition of Remote Attestation, we derive exact properties needed to attain it. We then translate them into architectural components, which we then map to a small set of hardware features that collectively achieve all required properties. We argue that the set of identified features form the minimal generic architecture for Remote Attestation. In the process, we remain agnostic with respect to the underlying hardware by making fewest possible assumptions about specific devices. We believe that the outcome of this effort is valuable, as it represents the first attempt to systematically explore the notion of Remote Attestation and to produce a light-weight blueprint that can be realized on wide range of devices, with minimal modifications. 


\section{RELATED WORK}

Software-Based Attestation. One early example of software-based attestation is Pioneer [30]. which does not rely on a secure co-processor or any specialized hardware. Pioneer computes a checksum of device memory using a function that includes side-effects (e.g., status registers) in its computation, such that any emulation of this function incurs a timing overhead sufficiently long to detect cheating. Attestation that relies on time-based checksums has also been adapted to embedded devices in [13], [16], [17], [27]-[29], [31]. However, some assumptions that form the basis for these solutions have been challenged [32] and several attacks on these (and similar) schemes have been proposed [4]. Moreover, Kovah et al. [14] showed that time-based attestation schemes may be vulnerable to Time Of Check, Time Of Use (TOCTOU) attack.

In general, all prior software-only solutions rely on strong assumptions on adversarial capabilities and only work if the verifier communicates directly to the prover, with no intermediate hops. While applicable to specific settings (e.g., attestation of computer peripherals), this approach is not viable for attestation performed over a network.

Static Root of Trust. An early example of a hardware-based mechanism is Secure Boot [2], which verifies system integrity at boot time. The root of trust is an immutable bootloader stored in ROM along with a public key. It verifies code signature and executes the code (i.e., boot) only if the signature is valid, thus authenticating code origin and integrity.

Trusted Platform Modules (TPMs) [35] are present in many commercial systems and used in several concrete architectures [13], [23]. TPM security is based on two properties: (1) Platform Configuration Registers (PCRs) are accessible only via a fixed API, and (2) PCRs that are reset only on boot, and each new measurement added (extended) to a PCR is added using a cryptographic hash of the previous PCR value and the new measurement. A TPM can sign a set PCRs that represent the load time state of the software that was executed on the computer. TPM is a static mechanism where the root of trust is the BIOS that performs the very first extension upon boot.

Dynamic Root of Trust. A dynamic root of trust provides a mechanism that can be used to perform an attestation dynamically, i.e., on the current state of the software. This requires additional features present in extended trusted computing specifications [35] as well as by CPU and chipset support by major vendors (e.g., Intel TXT [10] and AMD SVM [1]).

Flicker [18] is an architecture that establishes dynamic root of trust on commodity computers. It uses CPU extensions to execute a Piece of Application Logic (PAL) on the prover. Execution of PAL is guaranteed even if the platform's BIOS, OS and DMA are all compromised.

There are several techniques for remote trust establishment [12], [19], [20], [22], [36] with underlying platforms range from Web servers to embedded systems.

Other Hardware-Based Techniques. SPM [34] is a recent hardware-based mechanism for process isolation that uses a special vault module bootstrapped from a static root of trust. The vault bootstraps the SPM-protected programs, which gain exclusive control over the protection of their own memory pages. SMART [7] is a hardware-based scheme for establishing a dynamic root of trust in embedded devices. Its focus is on lowend microcontrollers (MCUs) that lack sophisticated features, such as specialized memory management or protection features. SMART requires small changes to the MCUs with limited hardware impact. Noorman et al. [21] describe a hardware architecture that provides a dynamic root of trust as well as other features, such as application isolation and authentication. Datta et al. [6] proposed LS2 - a logic for secure systems relying on a TPM. LS2 is used to describe attestation protocols standardized by the TCG, without providing a definition of attestation. In contrast, our goal is to describe the requirements at a lower level, without assuming the presence of a TPM or a similar device.

Finally, [24] is a ROM-based technique that fills the prover's entire memory with random data to ensure absence of malicious code. Although it involves minimal hardware requirements, this technique is limited to one-hop attestation since it lacks the means to authenticate a remote prover.

\section{Remote Attestation}

We use the term Remote Attestation to denote a protocol, whereby a challenger (Chal) verifies the internal state of another device called a prover (Prov). This protocol is performed remotely, i.e., over a network. The goal of the protocol is to allow an honest (not compromised) Prov to create an authentication token, that convinces Chal that the former is in some well-defined (expected) state. Whereas, if Prov has been compromised and its state has been modified, the authentication token must reflect this. We begin by defining the "remote attestation protocol".

Definition 1 (Remote Attestation Protocol): A protocol $\mathcal{P}$ comprised of the following components:

- Setup $\left(1^{\kappa}\right)$ - a probabilistic algorithm that, given a security parameter $1^{\kappa}$, outputs a long-term key $k$;

- Attest $(k, s)$ - a deterministic algorithm that, given a key $k$ and device state $s$, outputs an attestation token $\alpha$;

- $\operatorname{Verify}(k, s, \alpha)$ - a deterministic algorithm that, given a key $k$, a device state $s$ and an attestation token $\alpha$, outputs 1 iff $\alpha$ corresponds to $s$, i.e., iff Attest $(k, s)=\alpha$, and outputs 0 otherwise.

At the time of attestation, Prov's state $s=\left(s_{\text {Chal }}, s_{\text {Prov }}\right)$ consists of two parts: (1) $s$ Chal provided by Chal, e.g., a nonce, and (2) $s$ Prov that reflects the rest of Prov's state.

Next we define a game between Chal and Prov that leads to the definition of security for a remote attestation protocol.

Game 1 (Att-Forgery ${ }_{\text {Chal,Prov }}(\kappa)$ ): Chal running $\mathcal{P}$ interacts with Prov as follows:

1) Chal runs $k \leftarrow \operatorname{Setup}\left(1^{\kappa}\right)$ and outputs $s_{\text {Chal }}$ to Prov.

2) Prov is given oracle access to Attest. Specifically, Prov is allowed to adaptively submit $q$ device states $\left\{s_{1}, \ldots, s_{q}\right\}$. 
For each $s_{i} \neq\left(s_{\text {Chal }}, s_{\text {Prov }}\right)$, Prov receives the corresponding token $\alpha_{i}$.

3) Eventually, Prov outputs $\alpha$; the game outputs 1 iff $\operatorname{Verify}(k, s, \alpha)=1$, i.e., iff $\alpha$ corresponds to $s=$ $\left(s_{\text {Chal }}, s_{\text {Prov }}\right)$.

An honest Prov can trivially create $\alpha$ using Attest $(k, s)$. Whereas, if Prov has been compromised, its $s_{\text {Prov }}$ has changed and it must attempt to simulate the operation of Attest. This security game bears some resemblance to a MAC-Forge game [3]. Section VI-A discusses the relationship between remote attestation and MACs.

We now define our security notion, based on Game 1.

Definition 2 (Att-Forgery security): A remote attestation protocol $\mathcal{P}=$ (Setup, Attest, Verify) is Att-Forgery-secure if there exist a negligible function negl, such that, for any probabilistic polynomial time prover Prov and sufficiently large $\kappa$, it holds that: $\operatorname{Pr}\left[\right.$ Att-Forgery $\left.{ }_{\text {Chal,Prov }}(\kappa)=1\right] \leq \operatorname{negl}(\kappa)$

To simplify the notation, we say that $\mathcal{P}$ is a secure remote attestation protocol if $\mathcal{P}$ is Att-Forgery-secure. In Section IV, we identify properties required for Attest to provide secure remote attestation.

\section{A. System Model}

The central goal of any remote attestation protocol is to verify Prov's state. Successful protocol execution does not guarantee that Prov's entire system can be trusted or that the adversary can not modify Prov's state after attestation is completed.

We assume that Prov is a low-end embedded device with a single thread of execution, limited storage capacity and a low general complexity. Although our definition of Att-Forgerysecurity is valid for any device, its motivation is strongest for low-cost platforms where adding secure hardware components (e.g, a TPM [35]) would be too costly.

We make no assumptions about Chal. In particular, a malicious Chal can perform a Denial-of-Service (DoS) attack by forcing Prov to take part in the remote attestation protocol at will. Our security model is focused on a possibly malicious Prov and protection of Prov against DoS attacks is not a primary goal. Chal authentication, as well as replay and DoS mitigation is discussed in [9]. In the rest of this paper, we assume that Chal is honest. Note that $s_{\text {Chal }}$ (the part of Prov's state sent by Chal) can contain any information that Chal wants to be included in the computation of $\alpha$, e.g., a nonce, a sequence number or a timestamp.

We assume a reliable communication channel between Chal and Prov. We make no assumptions about its security, latency, packet routing or any other properties.

\section{B. Adversary Model}

We do not specify how Prov might be compromised; we assume that the adversary can do so at any time. Once Prov is compromised, we use the term prover to mean the device itself and the term adversary to reflect the adversary's presence on the prover. The distinction is relevant because, as we show below, in order to implement remote attestation securely, there must exist some secret quantity (i.e., a key) that the adversary can not access, even though it is in (almost) full control of Prov.

Once Prov is compromised, the adversary has full control over the code executing on the CPU. It can schedule interrupts at will, access all readable storage (including ROM) and modify to all writable storage. The only behavioral restrictions are those imposed by the hardware, e.g., the adversary can not write to ROM or force an interrupt if interrupts are disabled. We also assume that the adversary can not perform any hardware modifications to the prover, e.g., tamper with the digital logic, install a different CPU or add more memory.

Hardware side channels (e.g., measuring power consumption to infer bits of the key) or fault attacks (e.g., power glitches leading to incorrect execution of instructions) are very effective attacks on embedded systems. Such attacks are usually addressed via dedicated countermeasures (e.g., glitch sensors, dual rail logic), and are therefore out of the scope of this paper. Similarly, we assume that Attest is free of software vulnerabilities, implementation flaws and software (e.g., timing) side-channels.

\section{Properties ReQuired For Remote AtTestation}

We now discuss the necessary security properties for Attest to be used in secure remote attestation.

As follows from definition 2, Attest must satisfy the following: (1) only Attest can compute a valid token $\alpha$, and (2) $\alpha$ accurately captures the device state $s$, i.e., for any two states $s^{\prime} \neq s$, Attest $(k, s)=\operatorname{Attest}\left(k, s^{\prime}\right)$ with negligible probability. These observations lead us to two possible attack types:

Attack type 1: The adversary simulates Attest and correctly computes $\alpha$.

Attack type 2: Returned $\alpha$ does not correctly reflect $s$, i.e., the adversary escapes detection.

We now derive the complete set of security properties that Attest must satisfy to be Att-Forgery secure. Since $k$ is the only secret held by Prov, access to $k$ allows the adversary to simulate Attest, i.e., perform a type 1 attack by computing $\alpha$ without invoking the actual Attest. Therefore, we need the following property:

- Exclusive Access: Attest has exclusive access to $k$. This does not imply that the adversary can not learn some intermediate value that leaks information about $k$. Suppose that $\operatorname{Attest}(k, s)=\mathrm{HMAC}(k, s)=\mathrm{H}(k \oplus$ opad, $\mathrm{H}(k \oplus$ ipad,$s))$ and $k \oplus$ ipad is somehow leaked, e.g., remains in memory after computation of $\alpha$. This will allow the adversary to learn $k$, and use it to compute $\alpha$. Therefore, we need the following property:

- No Leaks: Attest leaks no function of $k$ other than $\alpha$. Another way of stating this property is that, after Attest completes, the entire state of Prov (except for $\alpha$ and $k$ itself) is statistically independent from $k$.

If the code comprising Attest is not protected, the adversary can modify it, e.g., by forcing it to output $k$, i.e., violate "exclusive access". Thus, an additional property is required: 
- Immutability: Attest code is immutable.

We stress that this property requires code to be executed in place from immutable memory. This is not always the case, e.g., when code is loaded from low-speed storage (e.g., FLASH) to high-speed memory, such as RAM or cache, before execution. The adversary could modify Attest after it is loaded into RAM, but before it is executed [26]. This is an instance of the wellknown Time-Of-Check-To-Time-Of-Use (TOCTTOU) attack, which can be prevented by a hardware signature check of the code, as in [10].

Taken together, three aforementioned properties are insufficient to protect Attest. Consider the case where memory is attested sequentially. As Attest reaches certain region $\mathrm{X}$ containing malicious code, it is interrupted, malicious code moves to an already verified memory location and original memory region $\mathrm{X}$ is restored before resuming attestation. Consequently, the adversary escapes detection. This is an example of a type 2 attack.

Note that checking memory in a pseudo-random fashion, as in [30], [31], does not solve the problem since the adversary can schedule an interrupt every time the next address is computed. Then, if the next address falls into the memory range occupied by malware, it moves its code fragment elsewhere and restores memory to its expected state. To prevent such attacks, we need another property:

- Uninterruptibility: Execution of Attest must be uninterruptible.

There remains a potential attack, despite all security properties described so far: the adversary can start execution in the middle of Attest, skipping important parts of the code. Suppose that, in the beginning of Attest, there is an instruction to enforce uninterruptible execution environment. If the adversary can start execution of Attest just after that instruction, the remainder of Attest would run in an interruptible manner, which prompts attacks of types 1 and 2, as shown earlier.

Assuming uninterruptibility of Attest, it might seem that, if the very first instruction of Attest loads $k$, even if the adversary invokes Attest in the middle, no information derived from $k$ can be learned and a valid $\alpha$ can not be computed. However, this argument is incorrect, for the following reasons:

First, in some instruction sets (e.g, Intel x86 [11]), skipping the first byte(s) of an instruction can lead to decoding a different instruction. Therefore, the adversary can jump into carefully selected locations in Attest changing its semantics in an unintended manner. Naturally, we prefer not to rely on features of a specific instruction set in stating general security properties. Second, the stated argument assumes that invoking Attest in the middle precludes the adversary from reading $k$. However, the adversary might be able to perform a returnoriented programming (ROP) [5], [15], [25] attack, as follows:

Jump into the beginning of some function within Attest. Since the jump instruction does not push a return address onto the stack, the stack will be "de-synchronized", i.e., the value specified in the stack by the adversary will become the return address of the function. When the function returns, it will jump to the address chosen by the adversary. This way, the adversary can cause Attest to jump anywhere within Attest code.

In general, if the adversary can influence control flow of Attest and alter its behavior, it can induce Attest to leak $k$ by reading it from restricted memory or by neglecting to erase it later. To prevent all such attacks, we need one final property:

- Controlled Invocation: Attest must only be invoked from its intended entry point.

In summary, the first three properties: exclusive access, immutability and no leaks, are necessary (but not sufficient) to prevent type 1 attacks. Whereas, the other two (uninterruptibility and controlled invocation) together enforce a semantically atomic execution of Attest. Though effective against some type 1 attacks, they mainly prevent type 2 attacks.

Under assumptions made above, we claim that any correctly implemented attestation protocol satisfying all five properties listed in this section is Att-Forgery-secure.

\section{A. Minimality of Properties}

We now argue that removing any postulated property leads to an insecure Attest. Note that each property is largely independent and eliminating any of them will make Attest vulnerable to the attack(s) described just above that property in the previous section. Specifically, if we were to omit:

- Exclusive Access to $k$ : the adversary would easily learn $k$.

- No Leaks: the adversary would learn information about $k$ that could lead to an advantage in computing a valid $\alpha$.

- Immutability: the adversary could change the code to move $k$ to unprotected memory.

- Uninterruptibility: the adversary could move malware around during attestation, which helps escape detection.

- Controlled Invocation: the adversary could invoke Attest anywhere, which might cause it to be interruptible and/or skip sanity checks on input parameters.

It thus becomes clear that any proper subset of the five properties is insufficient for secure remote attestation.

\section{Deriving Features from Properties}

In this section, we describe a combination of platform features that achieve the five security properties presented above. Our goal is to obtain a set of features that are both necessary and sufficient for remote attestation. We examine each property and identify features needed to attain it.

Exclusive Access to $k$. This is the most difficult property to impose on a low-end embedded device. There is no way to achieve it without some hardware support. If the underlying processor supports multiple privilege modes and a full-blown separation of memory for each process, we could use a privileged process to handle all computations that involve $k$. However, low-end processors generally do not offer such features. Our solution is to add a small hardware-based check that monitors the address bus and program counter (PC) and enforces $k$ only being accessible when PC is within Attest. We believe that this "custom" hardware check is unavoidable.

No Leaks. To make sure that no information related to (or derived from) $k$ is accessible when Attest completes we need 
a way to erase all intermediate values that depend on $k$, except the attestation token $\alpha$, when they are no longer needed.

Immutability. In order to make Attest immutable, we place it in ROM, which is available on most platforms. We consider ROM to be an inexpensive way to enforce immutability ${ }^{1}$. Attest needs to execute in-place from ROM.

Uninterruptibility. On a platform with a single thread of execution, the adversary can still regain control after invoking Attest by scheduling an interrupt. To enforce uninterruptibility, we need a way to disable (and enable) interrupts such that Attest will run from beginning to end. Moreover, the instruction to disable interrupts must itself be atomic. Otherwise, the adversary could interrupt this instruction and violate atomicity of Attest.

Controlled Invocation. As discussed earlier, we must enforce exclusive invocation of Attest from its very first instruction. Since there is no OS (or protected CPU) mode that enforces this on low-end devices, custom hardware is needed. As before, we use a small piece of custom hardware that implements the following logic: If the program counter (PC) is an address within the Attest code, other than the first instruction address, then the previous instruction must also be within Attest.

Secure Reset. Although Controlled Invocation precludes the adversary from jumping to the middle of Attest, in practice, there is no way to prevent this in an embedded system. The only option is to reset the device, whenever this property, or any other one, is violated. Such a reset must be triggered in hardware and, to prevent any sensitive memory contents to be leaked, all memory must be erased immediately after the device is reset.

Features described in this section form a set that is necessary and sufficient to support the security properties described in Section IV. We summarize them as:

- Custom hardware to enforce exclusive access to $k$.

- Reliable and secure memory erasure.

- Read-only-memory (ROM).

- Enable-interrupts and atomic disable-interrupts instructions.

- Custom hardware to enforce Attest being invokable only at the first instruction.

- Secure reset mechanism.

\section{A. Asymmetric Cryptography}

In terms of cryptographic primitives, our discussion has been focused on symmetric techniques. One interesting question is whether there are any benefits in using public key cryptography, i.e., digital signatures.

At the first glance, digital signatures would significantly complicate Attest code in terms of both size and execution speed. (Incidentally, the latter would increase the impact of

\footnotetext{
${ }^{1}$ There are several ways to achieve a similar goal, e.g., using an EEPROM or a Flash memory locked by a fuse. However, ROM is usually the simplest and cheapest to implement.
}

DoS attacks.) Also, instead of a shared $k$, the prover would need to store its private key $s k$ (in a secure location). However, none of this prompts the need for additional security features or components. On the other hand, as far as the verifier is concerned, $\alpha$ produced using a MAC is no less and no more secure than a digital signature computed over the same state. (Recall that our adversary model allows prover's compromise but not hardware attacks that could extract $k$ or $s k$.) We believe that the only potential advantage of digital signatures can be obtained if the application requirements of Remote Attestation include public verifiability of attestation tokens.

\section{DISCUSSION}

In this section we discuss some issues that were not adequately addressed earlier.

\section{A. Comparison with MAC}

Our definition of Remote Attestation functionality shares some features with the well-known and well-studied Message Authentication Code (MAC) primitive. Suppose that the legitimate prover has some secure hardware that can compute both MACs and attestation tokens. Furthermore, assume that the adversary can interrupt secure hardware execution. Whenever the verifier sends the challenge that includes $a, n$ and a nonce, along with expected memory contents in memory range $[a, a+n)$, the prover sends replies with: the challenge, its MAC and $\alpha$. Suppose that adversarial code resides in memory region $[a+n / 2, a+n)$. When MAC (or Attest) finishes computation for $[a, a+n / 2)$, the adversary interrupts the secure hardware, moves outside that range and restores all memory $[a+n / 2, a+n)$ to original contents. In this scenario, both MAC and $\alpha$ are computed correctly, as:

- The verifier believes that MAC is computed by the genuine prover.

- The verifier can not assert absence of adversarial code in memory range $[a, a+n)$ at attestation time.

This situation illustrates that uninterruptibility is not essential for MAC computation, whereas, it is essential to the security of remote attestation.

\section{B. Comparison with Secure Hardware}

While deconstructing our definition of remote attestation into properties, and mapping them to features, we described a mixed hardware-software system. Another option would be to design a purely hardware component that computes Attest atomically. This prompts the questions: Would a design based on a single piece of secure hardware require fewer security properties?

First, we only claim minimality of security properties that are quite independent of the specific architecture, rather than minimality of security features that are architecture-specific.

Second, we need to consider what security properties must be satisfied by the secure hardware component itself. In particular, this component would still have to satisfy all five security properties described earlier. 


\section{Untampered Execution Environment}

Attest does not automatically set up an untampered execution environment. However, it runs uninterrupted and authenticity of $\alpha$ guarantees absence of adversarial code in state $s$, at attestation time. We can take advantage of these properties to set up an untampered execution environment as follows.

Suppose that Attest disables interrupts during execution, as in Section V. First, the verifier sends the challenge that includes the nonce, the code to be executed, and (optionally) the expected dynamic environment state, i.e., stack memory location, global configuration variables, etc. Then, prover runs Attest uninterrupted: it computes $\alpha$, returns it to verifier, checks that the start address of the code is outside Attest and, if so, Attest immediately hands over control to the received code. Thus, when verifier receives a valid $\alpha$, it learns that the code it sent was executed uninterrupted in an untampered execution environment. This approach is similar to SMART [7].

\section{CONCLUSIONS AND FUTURE WORK}

This paper provided an in-depth systematic treatment of Remote Attestation and defined a new security notion for remote attestation protocols. Using this notion, we identified the necessary and sufficient properties needed for a device to support secure remote attestation. We then mapped these properties into a minimal collection of hardware and software components that collectively yield a secure attestation primitive.

Due to space limitations, this paper omits many interesting aspects of our work. The extended version of this paper [9] presents a protocol that uses the primitive derived above to achieve secure remote attestation. It also includes an analysis of a recent technique (SMART [7]) and identifies some surprising vulnerabilities. [9] also extensively discusses issues surrounding verifier authentication and DoS mitigation.

This work represents the first step towards a systematic study of Remote Attestation. There remain some important issues and questions for future work. Although we argued that identified properties and derived components collectively represent a minimal architecture for Remote Attestation, there could be other sets of components that also achieve minimality. We plan to further investigate this and implement the proposed architecture on several commodity platforms, possibly using public key signatures as an alternative to MACs. Finally, future work will include methods for automated verification of such properties on actual implementations.

\section{REFERENCES}

[1] Advanced Micro Devices. AMD, Secure Virtual Machine Architecture Reference Manual. Publication No. 33047, Revision 3.01, May 2005.

[2] W. A. Arbaugh, D. J. Farbert, and J. M. Smith. A secure and reliable bootstrap architecture. IEEE $S \& P, 1997$.

[3] M. Bellare, J. Kilian, and P. Rogaway. The security of the cipher block chaining message authentication code. Journal of Computer and System, 839:1-36, 2000.

[4] C. Castelluccia, A. Francillon, D. Perito, and C. Soriente. On the difficulty of software-based attestation of embedded devices. ACM CCS, 2009.

[5] S. Checkoway, L. Davi, A. Dmitrienko, and A.-r. Sadeghi. ReturnOriented Programming without Returns. ACM CCS, 2010.

[6] A. Datta, J. Franklin, D. Garg, and D. Kaynar. A logic of secure systems and its application to trusted computing. In IEEE $S \& P, 2009$.
[7] K. E. Defrawy, A. Francillon, D. Perito, and G. Tsudik. SMART: Secure and Minimal Architecture for (Establishing Dynamic) Root of Trust. In NDSS, 2012.

[8] N. Falliere, L. O. Murchu, and E. Chien. W32.Stuxnet Dossier. Symantec, October 2010.

[9] A. Francillon, K. Rasmussen, Q. Nguyen, and G. Tsudik. Systematic treatment of remote attestation. In Cryptology ePrint Archive: Report 2012/713, 2012.

[10] Intel Corporation. Intel Trusted Execution Technology (Intel TXT) Software Development Guide, 2009. doc: 315168-006.

[11] Intel Corporation. Intel 64 and IA-32 Architectures Software Developer's Manual, March 2012.

[12] R. Kennell and L. H. Jamieson. Establishing the Genuinity of Remote Computer Systems. In USENIX Security Symposium, 2003.

[13] C. Kil, E. C. Sezer, A. M. Azab, P. Ning, and X. Zhang. Remote attestation to dynamic system properties: Towards providing complete system integrity evidence. IEEE/IFIP DSN, 2009.

[14] X. Kovah, C. Kallenberg, C. Weathers, A. Herzog, M. Albin, and J. Butterworth. New Results for Timing-Based Attestation. In IEEE $S \& P, 2011$.

[15] S. Krahmer. x86-64 buffer overflow exploits and the borrowed code chunks exploitation technique. Technical report, SUSE, September 2005.

[16] Y. Li, J. M. McCune, and A. Perrig. SBAP: Software-Based Attestation for Peripherals. TRUST, 2010.

[17] Y. Li, J. M. McCune, and A. Perrig. VIPER: Verifying the Integrity of PERipherals Firmware. In ACM CCS, 2011.

[18] J. M. McCune, B. J. Parno, A. Perrig, M. K. Reiter, and H. Isozaki. Flicker: An Execution Infrastructure for TCB Minimization. In EuroSys, 2008.

[19] J. M. McCune, B. J. Parno, A. Perrig, M. K. Reiter, and A. Seshadri. How low can you go?: recommendations for hardware-supported minimal TCB code execution. ACM ASPLOS, 2008.

[20] C. Nie. Dynamic root of trust in trusted computing. TKK T1105290 Seminar on Network Security, 2007.

[21] J. Noorman, P. Agten, W. Daniels, R. Strackx, A. Van Herrewege, C. Huygens, B. Preneel, I. Verbauwhede, and F. Piessens. Sancus: Low-cost trustworthy extensible networked devices with a zero-software trusted computing base. In 22nd USENIX Security symposium. USENIX Association, August 2013.

[22] B. J. Parno, J. M. McCune, and A. Perrig. Bootstrapping Trust in Commodity Computers. In IEEE S\&P, 2010.

[23] S. Pearson, M. C. Mont, and S. Crane. Persistent and Dynamic Trust: Analysis and the Related Impact of Trusted Platforms. Security, 3477, 2005.

[24] D. Perito and G. Tsudik. Secure Code Update for Embedded Devices via Proofs of Secure Erasure. In Proceedings of the 15th European conference on Research in computer security (ESORICS), 2010.

[25] R. Roemer, E. Buchanan, H. Shacham, and S. Savage. Return-oriented programming: Systems, languages, and applications. Manuscript, V, 2009.

[26] R. Sailer, X. Zhang, T. Jaeger, and L. van Doorn. Design and implementation of a TCG-based integrity measurement architecture. In USENIX Security Symposium, 2004.

[27] A. Seshadri, M. Luk, and A. Perrig. SAKE: Software attestation for key establishment in sensor networks. Ad Hoc Networks, 9(6), 2008.

[28] A. Seshadri, M. Luk, A. Perrig, L. V. Doorn, and P. Khosla. SCUBA: Secure Code Update By Attestation in sensor networks. In ACM WiSec, 2006.

[29] A. Seshadri, M. Luk, A. Perrig, L. van Doorn, and P. Khosla. Using FIRE \& ICE for Detecting and Recovering Compromised Nodes in Sensor Networks. Technical Report December 2004, DTIC Document.

[30] A. Seshadri, M. Luk, E. Shi, A. Perrig, L. Van Doorn, and P. Khosla. Pioneer: verifying code integrity and enforcing untampered code execution on legacy systems. ACM SIGOPS OSRw, 39(5), 2005.

[31] A. Seshadri, A. Perrig, L. Van Doorn, and P. Khosla. SWATT: softwarebased attestation for embedded devices. In IEEE $S \& P, 2004$.

[32] U. Shankar, M. Chew, and J. D. Tygar. Side effects are not sufficient to authenticate software. USENIX Security Symposium, 8(3), May 2004.

[33] S. W. Smith. Outbound Authentication for Programmable Secure Coprocessors. International Journal of Information Security, 3(1), 2004.

[34] R. Strackx, F. Piessens, and B. Preneel. Efficient Isolation of Trusted Subsystems in Embedded Systems. SecureComm, 2010.

[35] Trusted Computing Group. TPM Main Specification Level 2 Version 1.2.

[36] Q. Yan, J. Han, Y. Li, and R. Deng. A software-based root-of-trust primitive on multicore platforms. In ACM ASIACCS, 2011. 